\title{
Lexical Specificity Training Effects in Second Language Learners
}

\author{
Caressa Janssen, ${ }^{a}$ Eliane Segers, ${ }^{a}$ James M. McQueen, ${ }^{a, b, c, d}$ \\ and Ludo Verhoeven ${ }^{a}$ \\ ${ }^{a}$ Radboud University, ${ }^{b}$ Max Planck Institute for Psycholinguistics, 'Donders Institute for Brain, \\ Cognition and Behaviour, and ${ }^{\mathrm{d} D}$ Donders Institute for Brain, Cognition and Behaviour
}

Children who start formal education in a second language may experience slower vocabulary growth in that language and subsequently experience disadvantages in literacy acquisition. The current study asked whether lexical specificity training can stimulate bilingual children's phonological awareness, which is considered to be a precursor to literacy. Therefore, Dutch monolingual and Turkish-Dutch bilingual children were taught new Dutch words with only minimal acoustic-phonetic differences. As a result of this training, the monolingual and the bilingual children improved on phoneme blending, which can be seen as an early aspect of phonological awareness. During training, the bilingual children caught up with the monolingual children on words with phonological overlap between their first language Turkish and their second language Dutch. It is concluded that learning minimal pair words fosters phoneme awareness, in both first and second language preliterate children, and that for second language learners phonological overlap between the two languages positively affects training outcomes, likely due to linguistic transfer.

Keywords lexical specificity; phonological awareness; intervention; second language learning; linguistic transfer

\section{Introduction}

Large groups of children around the globe start formal education in a language other than their home language. These sequential bilinguals may experience slower vocabulary growth in their second language (L2), relative to their native-speaking classmates, subsequently leading to disadvantages in literacy

\footnotetext{
The elicitation instruments used for this study can be accessed by readers in the IRIS digital repository (http://www.iris-database.org).

Correspondence concerning this article should be addressed to Caressa Janssen, Behavioral Science Institute, Radboud University, Montessorilaan 3, P.O. Box 9104, 6500 HE Nijmegen, Netherlands. E-mail: c.janssen@pwo.ru.nl
} 
acquisition (Bialystok, Luk, Peets, \& Yang, 2010; Kieffer, 2008; Leseman, 2000; Verhoeven \& Vermeer, 2006a). We asked whether these disadvantages can in part be overcome through training bilingual children about the detailed phonological makeup of spoken words. Training lexical specificity, defined as the richness and specificity of, and distinctness between, phonological representations in the emerging mental lexicon, has been found to facilitate phonological awareness in the first language (L1) of preliterate children (Van Goch, McQueen, \& Verhoeven, 2014). In the Van Goch et al. study, 4-year-old monolingual Dutch children were presented in a word learning game with new words with only minimal acoustic phonetic differences. These children showed gains in their detailed phonological knowledge as a result of training, demonstrating increased rhyme awareness, an aspect of phonological awareness. Because phonological awareness is an important precursor for literacy development (Melby-Lervåg, Halaas Lyster, \& Hulme, 2012), lexical specificity training may thus foster reading acquisition in the L1. The focus of the present study was on whether lexical specificity training can also facilitate L2 phonological awareness in preliterate bilingual children. Furthermore, we asked to what extent linguistic transfer from L1 to L2 plays a role in lexical specificity training and hence in the development of phonological awareness.

\section{Lexical Specificity}

Around the age of 1 year, children's lexical development is given a boost as they learn to distinguish word meanings based on the phonetic differences between speech sounds and to create phonological representations of words in memory (Werker, Byers-Heinlein, \& Fennell, 2009). Phonological representations can be described as "a characterization of words as composed of discrete phonological categories interpreted within a framework in which single-category differences signal lexical distinctions" (Yoshida, Fennell, Swingley, \& Werker, 2009, p. 416). These phonological representations are holistic to begin with, but become more detailed and segmental as vocabulary grows, which is referred to as lexical restructuring (e.g., Gerken, Murphy, \& Aslin, 1995; Goswami, 2000; Metsala \& Walley, 1998; Morais, 2003; Thiessen, 2007). With only a few words in the vocabulary, holistic representations would be sufficient. For example, the word 'bear' can be easily distinguished from the word 'fence'. However, as vocabulary size increases, more fine-grained phonological representations are necessary for listeners to be able to distinguish words based on only minimal acoustic-phonetic differences. 
With increasing numbers of words in the mental lexicon, encountering minimal pairs - pairs of words that differ in only one acoustic-phonetic featurebecomes more likely. For example, identifying the words 'bear' and 'pear' as two different words with different meanings is difficult as the phonemes $/ \mathrm{b} /$ and $/ \mathrm{p} /$ differ only in voicing. The quality or distinctness of phonological representations (i.e., lexical specificity) thus supports spoken word recognition (Metsala \& Walley, 1998). Although lexical representations appear to be phonologically highly specific much earlier in development than initially proposed by Metsala and Walley (1998), that is, in toddlers under the age of two (e.g., Swingley \& Aslin, 2000; White \& Morgan, 2008; Yoshida et al., 2009), it remains the case that increases in vocabulary size in children of any age are also likely to trigger increases in lexical specificity.

The degree of lexical specificity may influence several kinds of phonological processes, including speech decoding. For example, in order to understand speech, the acoustic information in the continuous speech stream needs to be decoded (categorized) into discrete, meaningful units, such as phonemes and, hence, spoken words. Increases in lexical specificity could help children improve their speech decoding ability by encouraging them to focus on dimensions of contrast that are critical for the differentiation of words and therefore to be distracted less by dimensions that are not important for this task, such as inter- and intraspeaker variability (White \& Morgan, 2008).

Lexical specificity has also been found to influence phonological awareness (Elbro, Borstrøm, \& Petersen, 1998; Metsala \& Walley, 1998; Walley, Metsala, \& Garlock, 2003). Being phonologically aware means being sensitive to, or explicitly aware of, the phonological structure of words and being able to manipulate this structure (Torgesen, Morgan, \& Davis, 1992). Phonological awareness is an important factor in word learning (Hu, 2003; Torgesen et al., 1992) and is one of the strongest predictors of later reading skills and generally of school success, across languages (Castles \& Coltheart, 2004; Melby-Lervåg et al., 2012; Siok \& Fletcher, 2001). Phonological awareness develops across languages, including Dutch, from larger to smaller sound units, that is, from syllables to rhymes to phonemes (Carroll, Snowling, Hulme, \& Stevenson, 2003; Mark, Müller-Myhsok, Schulte-Körne, \& Landerl, 2014; Patel, Snowling, \& De Jong, 2004; Vloedgraven \& Verhoeven, 2007; Ziegler \& Goswami, $2005)$. In order to manipulate phonological structures in more and more detail, specific phonological representations of words may be required (De Cara \& Goswami, 2003; Elbro et al., 1998; Garlock, Walley, \& Metsala, 2001).

Recently, Van Goch et al. (2014) found that word learning focusing on lexical specificity enhanced rhyme awareness in monolingual Dutch kindergartners. 
Twenty-four quadruplets of monosyllabic Dutch words with corresponding pictures were presented to 4-year-olds in a word-learning game. The quadruplets consisted of two target words that were unfamiliar to the children (e.g., aar and aal, "ear of corn" and "eel"), one familiar control word (e.g., aap "monkey") and one unfamiliar control word (e.g., aas "bait"). All stimulus words, unfamiliar and familiar (control) words, were selected based on the Basiswoordenlijst Amsterdamse Kleuters [Basic Vocabulary of Kindergartners in Amsterdam] (Mulder, Timman, \& Verhallen, 2009). Stimulus words were considered as familiar if they appeared on this list and as unfamiliar if they did not appear on this list. The target words in each quadruplet formed minimal pairs. Control words differed in two acoustic-phonetic features with the target words. Van Goch et al. showed that learning new words successfully was dependent on gains in detailed phonological knowledge during training, which in turn led to improvement on rhyme awareness.

The question then emerges whether lexical specificity training in L2 could enhance speech decoding and/or phonological awareness in bilingual children. Whereas monolingual children have to deal with only one form of linguistic input, bilingual children are confronted with two. This has consequences for both L1 and L2 development in bilingual children. Initially, L1 acquisition of sequential bilinguals (i.e., those who are exposed to their L2 later in childhood) is expected to match L1 acquisition of monolingual children, but from the moment a majority L2 is introduced, sequential bilinguals show different patterns of L1 development. For example, previous research has documented continued growth of the L1, although at a slower pace than in monolingual children (e.g., Jia, Strange, Wu, Collado, \& Guan, 2006), levelling off in L1 proficiency (e.g., Kan \& Kohnert, 2005), and even regression of L1 ability (e.g., Francis, 2005). With respect to L2 development, consistent growth of L2 vocabulary patterns has been found across studies (August et al., 2006; Bialystok et al., 2010; Kan \& Kohnert, 2005; Kohnert, Windsor, \& Ebert, 2009; Uchikoshi, 2006). However, at the beginning of primary school, the L2 vocabulary size of children from cultural minorities in the Netherlands who learn L2 Dutch, the focus group of the current study, is smaller than the estimated L1 vocabulary size of age-matched Dutch monolingual children. These differences are also likely to remain constant as bilinguals grow older (Verhoeven \& Vermeer, 2006a).

There are additional differences in the L1 and L2 developmental patterns of sequential bilinguals. For example, lexical-semantic information is crosslinguistically distributed, that is, some concepts are lexicalized only in the L1, whereas other concepts are lexicalized only in the L2 (Kan \& Kohnert, 2005). Moreover, because sequential bilinguals lack early experience with the 
phonological structure of their L2, phonological representations of words in this language may be less specific than phonological representations of words in their L1 and in the L1 of monolingual children. Furthermore, sequential bilinguals may categorize L2 sounds according to the representations they have in their L1 (Best \& McRoberts, 2003). Existing linguistic knowledge may have to be reorganized in order to specify L2 phonological representations to the same extent as L1 phonological representations (Carroll, 2008). Because of these differences in L1-L2 developmental patterns, lexical specificity training could have different effects in bilingual versus monolingual children.

\section{Lexical Specificity Training and Phonological Processes}

Lexical specificity training may have several benefits for bilingual children's speech decoding. As results of behavioral and neurocognitive experiments have shown (see Kuhl, 2004, for an overview), young infants have the ability to distinguish speech sounds independently of the language spoken in their environment. However, perception of speech becomes attuned to the language that is spoken in that environment as a function of language experience during the first year of life (Cheour et al., 1998). L2 lexical specificity training may therefore enhance speech decoding more in bilingual children than in monolingual children. This is because monolingual children are likely to be more familiar with all the phonetic contrasts encountered in the training than bilingual children, which is consistent with the results of Van Goch et al. (2014) who found no effect of lexical specificity training on speech decoding for monolinguals. From a very young age onwards, monolinguals are confronted with speech contrasts that are important for distinguishing phonological representations of words in their language, whereas bilingual children still need to find out which speech contrasts are important to distinguish in their L2. Lexical specificity training in their L2 could help them with this task.

Lexical specificity training may also aid bilinguals' phonological awareness. For example, Treiman and Zukowski (1996) found that having more specific lexical representations makes it easier to make rhyme judgments. Moreover, lexical specificity training has already been found to enhance rhyme awareness in monolingual preliterate children (Van Goch et al., 2014). Awareness of rhyme structure (as measured in a rhyme awareness task) is one of the earliest forms of phonological awareness to develop (Carroll et al., 2003; Vloedgraven \& Verhoeven, 2007; Ziegler \& Goswami, 2005). Phoneme awareness (e.g., as measured in a phoneme blending task), which develops later than the awareness of rhyme structure, has also been found to predict later printed word recognition 
skills across languages (Muter, Hulme, Snowling, \& Stevenson, 2004; Mark et al., 2014). Gains in detailed phonological knowledge via lexical specificity training may foster phoneme awareness which can in turn simplify the task of word identification, such as when children hear $/ \mathrm{p} / / \mathrm{o} / / \mathrm{p} /$ and have to identify it as pop, meaning "doll” (Walley et al., 2003).

Effects of lexical specificity training on phonological awareness may differ between monolingual and bilingual children. On the one hand, L2 lexical specificity training could enhance phonological awareness less in bilingual than in monolingual children. Because of the smaller L2 vocabulary size of sequential bilingual children and differences in lexical development compared to monolingual children, bilingual children may have more difficulty with learning new phonological representations, in turn leading to less enhancement of phonological awareness (Goswami, 2000; Metsala, 1999). On the other hand, L2 lexical specificity training could enhance phonological awareness in bilingual children to the same extent as, or maybe even to a greater extent than, in monolingual children. Several studies (e.g., Bialystok, Majumber, \& Martin, 2010; Campbell $\&$ Sais, 1995) have found beneficial effects of phonological awareness from L2 exposure at a preliterate stage. Experience with two language systems and frequent attention to the phonotactic aspects of language would lead to relatively high levels of phonological awareness and advantages in learning about spoken language. Training lexical specificity in the L2 could thus increase experience with the phonological structure of the L2 and stimulate L2 vocabulary development, facilitating the development of phonological awareness.

\section{Lexical Specificity Training and Linguistic Transfer}

Studying a bilingual population not only offers us insights into L2 learning, it also enables us to investigate possible linguistic transfer from one language to the other (e.g., Hernandez, Li, \& MacWhinney, 2005). Different forms of transfer can occur. Learning an L2 can influence the L1, especially in the first 5 years of life, since these young children's L1 phonology and speech production capabilities are still developing (Gildersleeve-Neumann, Pena, Davis, \& Kester, 2009; Goldstein \& Bunta, 2012; Kan \& Kohnert, 2012). Also, bits of one language can slip into the output of the other language being spoken or written (Grosjean, 2012; Kohnert \& Bates, 2002). Finally, children's L1 can influence acquisition of the L2, for example, as mentioned earlier, bilinguals may categorize L2 sounds according to the representations they have in their L1 (Carroll, 2008; Navarra, Sebastián-Gallés, \& Soto-Faraco, 2005). Results of several studies provide evidence for transfer from the L1 to the L2 in emerging 
literacy skills in young bilingual children. Gottardo, Yan, Siegel, and WadeWoolley (2001), for example, found that phonological processing skills in children's L1 are related to phonological processing skills in their L2. Furthermore, Dickinson, McCabe, Clark-Chiarelli, and Wolf (2004) showed preschool phonological awareness skills to transfer from L1 to L2 in low-income SpanishEnglish bilingual children. In a longitudinal study, Verhoeven (2007) examined L2 development in Turkish-Dutch bilingual kindergarten children and found that, over time, the bilingual children performed similarly to native speakers in many Dutch language proficiency tests. However, residual asymmetries showed that sometimes children's L2 skills were still dependent on their L1 skills. Furthermore, children's level of proficiency in both the L1 and the L2 was found to predict variation in phonological awareness at the end of kindergarten.

According to the Competition Model (MacWhinney, 2004), the level of interaction between L1 and L 2 depends on the degrees of similarity between the languages and on language users' levels of proficiency in them. Each language has its own phonological makeup, and languages differ in the degree to which they have overlapping phonological structures. This overlap in phonological structure, so-called typological closeness, has been shown to facilitate linguistic transfer (e.g., Cenoz, 2001; Jarvis \& Odlin, 2000; Odlin, 1989; Selinker \& Lakshmanan, 1993), even at high levels of language proficiency (de Bot, 1992; Poulisse, 1990). It can therefore be predicted that phonological overlap between the L1 and the L2 in the words used in lexical specificity training may promote linguistic transfer and enhance training outcomes in bilingual children.

\section{The Current Study}

In summary, children who start formal education in an L2 may experience slower vocabulary growth in that language, which could subsequently lead to disadvantages in literacy acquisition. These disadvantages may in part be overcome by teaching children about the detailed phonological makeup of spoken words (i.e., through lexical specificity training). Training to make new lexical representations more specific enhances phonological awareness in monolingual children (Van Goch et al., 2014), phonological awareness being a strong predictor of later reading skills. However, it is still unclear, first, whether gains in speech decoding and/or gains in phonological awareness can be made in the case of L2 lexical specificity training for bilingual children, similar to L1 lexical specificity training for monolingual children and, second, to what extent linguistic transfer will have an impact on training results. In the present intervention study, we addressed these two questions by examining the effects 
of lexical specificity training on speech decoding and phonological awareness in 4-year-old L2-Dutch children in the Netherlands. For the training, we followed the same protocol as that in the study of Van Goch et al. The role of linguistic transfer was explored by making a distinction between words with phonemes that overlapped between L1 and L2 and those with phonemes that occur only in the L2.

It is important to note that a training protocol that has been successful in monolingual children may not necessarily have the same effects in bilingual children. Since the sequential bilingual children in the current study have a smaller L2 vocabulary size than the L1 vocabulary size of monolingual children, the bilingual children may be less familiar with the already-known words in the protocol than the monolingual children. This could attenuate training effects in bilingual children. Moreover, learning words in the L2 partly differs from learning words in the L1. Whereas monolingual children learn a word form and relate it to a concept over time (e.g., Bloom, 2000), for bilingual children, word learning largely involves mapping two word forms (in L1 and L2) onto a single concept (Kan \& Kohnert, 2008). There are individual differences in growth of novel word knowledge in L1 and L2, possibly due to differences in learning style, language learning environment and cultural differences (Kan, 2010). The current study could thus reveal whether the lexical specificity training protocol can be effective only in monolingual children or in both monolingual and bilingual children.

With regard to the first question, it was expected that training lexical specificity would foster speech decoding and phonological awareness in bilingual children. Vocabulary growth does not only contribute to phonological detail in word representations by providing contrasting phonemes, but also more sharply defines categories for individual phonemes themselves (Roberts, 2005). Learning new L2 words with only minimal differences in phonological structure during lexical specificity training may therefore simulate the effects of normal L2 vocabulary development in bilingual children, more specifically by triggering improvements in phonological skills, such as speech decoding and phonological awareness. With regard to the second question, it was expected that the degree of phonological overlap between L1 and L 2 in stimulus materials would have a positive effect on gains in speech decoding and phonological awareness in bilingual children. Similarity between the two languages in the lexical specificity training would allow linguistic transfer and lead to better training results (MacWhinney, 2004). This in turn could contribute to enhancement of speech decoding and phonological awareness. 


\section{Method and Materials}

\section{Participants}

A total of 32 L1-Dutch kindergarten children (15 boys, 17 girls) and 29 L2Dutch kindergarten children with Turkish as their first language (17 boys, 12 girls) participated (mean age L1-Dutch children at pretest: 56 months, range: 50-63 months, $S D=3.50$; mean age L2-Dutch children at pretest: 58 months, range 50-66 months, $S D=4.01$ ). The children were divided into an L1-Dutch experimental and control group and an L2-Dutch experimental and control group.

In the Netherlands, kindergarten is a 2-year program, prior to Grade 1, which starts in the year the child turns 4 . Children can start kindergarten on their fourth birthday, so most are in kindergarten for more than 2 years, but less than 3, frequently in mixed-age groups. In kindergarten, Dutch children develop phonological skills, such as rhyming, via songs, stories, and games. Although teachers also pay some attention to grapheme-phoneme correspondences, formal reading instruction via phonics teaching methods (as used in the Netherlands) does not start before Grade 1 . The children in our study were all in their 1st year of kindergarten and came from eight kindergarten groups from three primary schools, in three different cities distributed throughout the Netherlands. The parent(s) gave informed consent for the participation of their child.

In all schools, children and parents were obliged to communicate in Dutch with teachers and other children in the classroom. Ninety-four percent of the parents of the L1-Dutch children and 76\% of the parents of the L2-Dutch children responded to questions about SES and language use at home. Parental level of education was measured by making a distinction between high level ( 3 = higher professional education), intermediate level $(2=$ lower vocational education), and low level of education $(1=$ primary school). On average, parents of the L1-Dutch children were educated at an intermediate level (father: $M=2.43, S D=.50$, mother: $M=2.43, S D=.50$ ), parents of the L2-Dutch children were educated at a lower level (father: $M=1.77, S D=.75$, mother: $M$ $=1.91, S D=.75)$. The difference in educational level is significant, for both fathers, $t(50)=3.02, p=.004, d=.85$, and mothers, $t(48)=3.69, p=.001$, $d=1.06$.

Language use was measured by asking whether parents of the children were speaking only Turkish (1), mostly Turkish, sometimes Dutch (2), mostly Dutch, sometimes Turkish (3), only Dutch (4) or another language (5) to the child. In $96 \%$ of all L1-Dutch households (30) that responded to the questions, both 
parents spoke only Dutch to their child. In $2 \%$ of the households, both parents spoke mostly Dutch, sometimes another language to their child. In another $2 \%$ of the households, both parents spoke to their child in a Dutch dialect. In $50 \%$ of all L2-Dutch households (22) that responded to the questions, both parents spoke only or mostly Turkish to their child. In $36 \%$ of the households, one parent spoke mostly Turkish to his/her child, the other parent spoke mostly Dutch to his/her child. In $14 \%$ of the households, both parents spoke mostly Dutch, sometimes Turkish to their child.

\section{Basic Cognitive Skills}

Phonological Working Memory

Phonological working memory in Dutch was assessed with the subtest Geheugen [Memory] of the standardized Screeningstest voor Taal- en Leesproblemen [Diagnostic Test for Language and Literacy Problems] (Verhoeven, 2005). This test consisted of three parts. In the first part, the child was asked to repeat lists of increasing length, consisting of only monosyllabic words (12 items). The maximum length of these lists was seven words. The child received one point for each correctly repeated list of words. In the second part, the child was asked to repeat words in sentence contexts (12 items). The sentences increased in length, with a maximum of 17 words per sentence. The child received two points for each correctly repeated sentence. If the child made only one mistake, one point was given. If the child made more than one mistake, no points were given. When the child scored no points for four consecutive sentences, testing was stopped. In the final part, the child was asked to repeat 40 nonwords which were phonotactically legal in Dutch. Of these 40 nonwords, the first eight words consisted of one syllable, with the following four sets of eight words containing two, three, four, and five syllables each. The child received one point for each correctly repeated nonword. Testing was stopped after the child scored no points for five consecutive nonwords. The total test score was the total number of points collected in all three parts of the test, with a maximum score of 76 points (12 points in the first part, 24 points in the second part, 40 points in the last part).

\section{Rapid Naming}

The subtest Woordbenoemen [Word naming] of the standardized Screeningstest voor Taal - en Leesproblemen [Diagnostic Test for Language and Literacy Problems] (Verhoeven, 2005) was used to assess naming speed. From a sheet with four columns of thirty pictures, the child had to name as many pictures as possible correctly within one minute. The pictures represented five different 
objects (e.g., a comb, a duck, a pair of glasses, a house, and a shoe). Before the child started with the task, it was ensured that he/she was familiar with the pictures and that he/she could name them in Dutch. The total test score was the number of pictures named correctly within 1 minute.

\section{Receptive Vocabulary}

The receptive vocabulary test of the Taaltoets Alle Kinderen [Language Test for all Children] (Verhoeven \& Vermeer, 2006b) was used. Each item consisted of four pictures that were shown to the child. The meaning of one of the pictures was asked and the child had to point out the correct picture. A total of 52 items (42 nouns, 10 verbs) with increasing difficulty were presented. If the child did not respond correctly to five consecutive items, the task was ended. The total test score was the number of items responded to correctly. The L2-Dutch children also completed the receptive vocabulary test of the Toets Tweetaligheid [Test Bilingualism] (Verhoeven, Narain, Extra, Konak, \& Zerrouk, 1995) in Turkish. Again, each item consisted of four pictures that were shown to the child. The meaning of one of the pictures was asked in Turkish and the child had to point out the correct picture. A total of 40 items ( 25 nouns, 15 verbs) with increasing difficulty were presented. After the child did not respond correctly to five consecutive items, the task was ended. The total test score was the number of items responded to correctly.

\section{Phonological Skills}

Speech Decoding

The subtest Auditieve Discriminatie [Phoneme Discrimination] of the Screeningstest voor Taal - en Leesproblemen [Diagnostic Test for Language and Literacy Problems] (Verhoeven, 2005) was used to assess children's perception of minimal phonemic differences in monosyllabic words in Dutch (a speech decoding skill). Minimal word pairs, differing with respect to only one phoneme (e.g., val-wal "fall-quay") were presented auditorily to the child (two practice items, 30 test items). The child had to indicate whether the word pair consisted of the same two words or two different words. Before the task started, the experimenter checked that the child was familiar with the meaning of "the same" and "different" and made sure that the child knew these terms applied to the sound of the words, not the meaning.

\section{Phonological Awareness}

The children's phonological awareness was assessed using the rhyme awareness and the phoneme blending tasks of the Screeningsinstrument Beginnende 
Geletterdheid [Diagnostic Instrument for Emergent Literacy] (Vloedgraven, Keuning, \& Verhoeven, 2009). The rhyme awareness task targeted awareness of larger sound units (rhymes). The task involved two practice items and 15 test items. Each item consisted of three pictures (e.g., sok "sock", nek "neck", and hoek "corner") presented on a computer screen. The monosyllabic words depicted in the images were pronounced by a female voice during their presentation on the computer screen. Then the female voice pronounced a fourth word that rhymed with one of the three pictures on the screen (e.g., koek "cake", which rhymes with hoek). The child had to identify and press the correct picture. The total test score was the number of correctly identified pictures. The phoneme blending task targeted awareness of smaller sound units (phonemes). This task also involved two practice items and 15 test items. Each item consisted of three pictures (e.g., mier "ant", vuur "fire", muur "wall") presented on a computer screen. The monosyllabic words depicted in the images were pronounced by a female voice. Then the same voice pronounced the name of one of the pictures phoneme by phoneme. The child had to identify and press the correct picture. The total test score was the number of correctly identified pictures.

\section{Training}

\section{Lexical Specificity Training}

The lexical specificity training protocol was designed by Van Goch et al. (2014). In this protocol, the children were taught new words in pairs that were phonologically minimally different. The protocol was programmed in Presentation software (Neurobehavioral Systems, Albany) and presented on a computer screen. Twenty-four quadruplets of monosyllabic Dutch words with corresponding pictures were created for the present study. The quadruplets consisted of two unfamiliar minimal-pair target words differing in one acoustic-phonetic feature (e.g., vak-wak "section-ice hole"), one unfamiliar control word (e.g., rak "straight part of a river") and one familiar control word (e.g., pak "package"), with the control words different from the target words in two acousticphonetic features. The target and control words were selected from the Streeflijst voor 6-jarigen [Target List for 6-Year-Olds], based on familiarity ratings (Schaerlaekens et al., 1999). These ratings were the percentages of agreement among teachers about familiarity of words to 2nd-year preschoolers (6-yearolds). A word that did not occur on the list or one whose percentage was between 0 and 75 was considered unfamiliar and was selected as a target word or unfamiliar control word; a word with a percentage over 75 was considered as familiar and was selected as a familiar control word. 

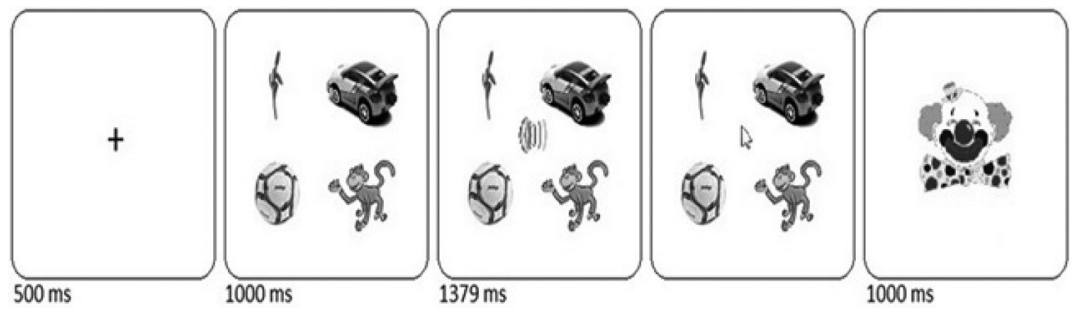

Figure 1 Sample trial sequence from the first block of lexical specificity training.

Half of the targeted minimal acoustic-phonetic distinctions occurred only in Dutch, whereas the other half occurred in both Dutch and Turkish. Compared with the Dutch phonological system, the Turkish one has only two distinctive levels of vowel height (high and low), there is no contrast between lax and tense vowels, and there are no original (not loaned) diphthongs (cf. Boeschoten \& Verhoeven, 1987). Also, the Dutch consonants /g/ and /w/ do not exist in Turkish, and consonant clusters do not occur at the beginning of words. Half of the quadruplets contained vowel distinctions and half of the quadruplets contained consonant distinctions. Finally, all quadruplets were matched for type of distinction (i.e., manner, place, or voice) and place of distinction (i.e., initial or final for consonants, and medial for vowels). In addition to the target and control words, filler words were used in the training. These were all highly familiar to the children and phonologically unrelated to the target and control words. The target words were recorded by one native Dutch female speaker and used as the target sound files in the training. For an overview of all the quadruplets used in the training, see Appendix S1 of the Supporting Information online.

The lexical specificity training consisted of a practice phase and a training phase. In each trial, four pictures were shown on a computer screen. Two of these pictures represented experimental items (target and control words) and the other two pictures represented filler items (e.g., a car and a ball), as shown in Figure 1. In total, 134 trials were included. The practice phase, with five practice trials, was used to familiarize the children with the training and to explain a strategy that could be used to find the correct answers (as explained below). The training phase consisted of two blocks of 48 experimental trials and one block of 24 experimental trials. In the first two blocks, each quadruplet of items appeared once; in the last block, each pair of target items appeared once (see Appendix S2 in the Supporting Information online). The quadruplets in the first two blocks and the pairs of target items in the last 
block were presented in a pseudo-randomized order. Also, the distinctions in segmental target (vowels vs. consonants), language (either Dutch or both Dutch and Turkish), stimulus type (manner, place or voice) and place (initial, medial, or final) were pseudo-randomized. There were a maximum of three trials of the same type in succession. Furthermore, nine filler trials were included. These trials were easier than the experimental trials; only pictures were shown that represented filler items. Filler trials were randomly distributed throughout the training phases, to keep motivation high.

On every trial, the child was asked an auditory question, "Wat is denk je een [TARGET]?" (What do you think is a [TARGET]?). Then the child had to press the picture that corresponded with the answer to the question. During the practice phase, the children were offered a strategy for finding the correct picture in each trial. They were told to consider the meaning of each picture on the screen, to rule out the familiar pictures first and select the picture they were (most) unfamiliar with. Using this process of elimination to select the only unknown picture as referent for the new word could help with completing the task successfully. Then, in Block 1 of the training phase, each member of a given novel minimal pair (target word 1 and target word 2) was combined with its familiar control word and two filler words. In Block 2, each target word was combined with its unfamiliar control word and two filler words. Finally, in Block 3, target words 1 and 2 were presented together, along with two filler words. In this last block, in half of the trials the children were asked to identify target word 1, and in the other half of the trials they had to identify target word 2.

Each trial started with presentation of a fixation cross (500 milliseconds), after which the four pictures were presented. After 1,000 milliseconds the auditory question was played (mean duration: 1,379 milliseconds) while the pictures remained on the screen. If the child pressed the correct picture, a picture of a clown appeared on the screen, providing positive feedback (1,000 milliseconds). If the child did not press the correct picture, no feedback was provided and the next trial started (see Figure 1 for a sample trial). Feedback for correct answers was provided in the practice and training phases. The training took about 15 minutes on average.

\section{Numeracy Training (Control)}

The control group received training with numeracy concepts that was similar to the lexical specificity training. The numeracy concepts in this training formed pairs (contrasts). Concepts were lowest/highest, smallest/biggest, shortest/longest, and least/most. Thirty different pairs were created. In each trial, 
one pair was presented on a computer screen. Two pictures were shown, for example, a picture of a house (lowest) and a picture of an apartment building (highest). Again, the child had to press the picture that corresponded to the answer in response to an auditory question (e.g., Wat is denk je het laagst? [What do you think is the lowest?]). Constraints for randomization, filler trials and procedure for providing feedback were similar to the lexical specificity training protocol. In total, 120 trials were included in the numeracy training; it took about 15 minutes on average.

\section{Procedure}

A pretest/posttest design was used. At pretest, children's basic cognitive skills (phonological working memory, naming speed, and receptive vocabulary, which was also measured in Turkish for the L2-Dutch children) were administered as control variables that were used to divide the children into four matched groups, namely, L1-Dutch experimental and control groups ( $n=16$ in each group), and L2-Dutch experimental and control groups ( $n=14$ and $n=15$, respectively). Then the rhyme awareness, phoneme blending, and phoneme discrimination tasks were administered. A few days after the pretest, the children in the experimental groups received the lexical specificity training and the children in the control groups received the numeracy training. The lexical specificity training was presented as a word learning game. One week later, a posttest took place, with the rhyme awareness, phoneme blending, and phoneme discrimination tasks administered again. The children were tested individually in a quiet room at their primary school. The pretest took two 30-minute periods with a break in between, and the posttest took 30 minutes. All children were tested in Dutch (pretest, lexical specificity training/numeracy training, and posttest) by the same female native Dutch speaker (the first author). The Turkish vocabulary test was administered by a female native Turkish speaker (a trained research assistant).

\section{Results}

\section{Descriptive Statistics}

First, we compared the four groups (experimental vs. control crossed by L1Dutch and L2-Dutch) for the control measures (rapid naming, phonological working memory, receptive vocabulary in Dutch, receptive vocabulary in Turkish) and the experimental measures prior to the intervention (pretest scores on rhyme awareness, phoneme blending, and phoneme discrimination). Table 1 summarizes descriptive statistics for these measures. 


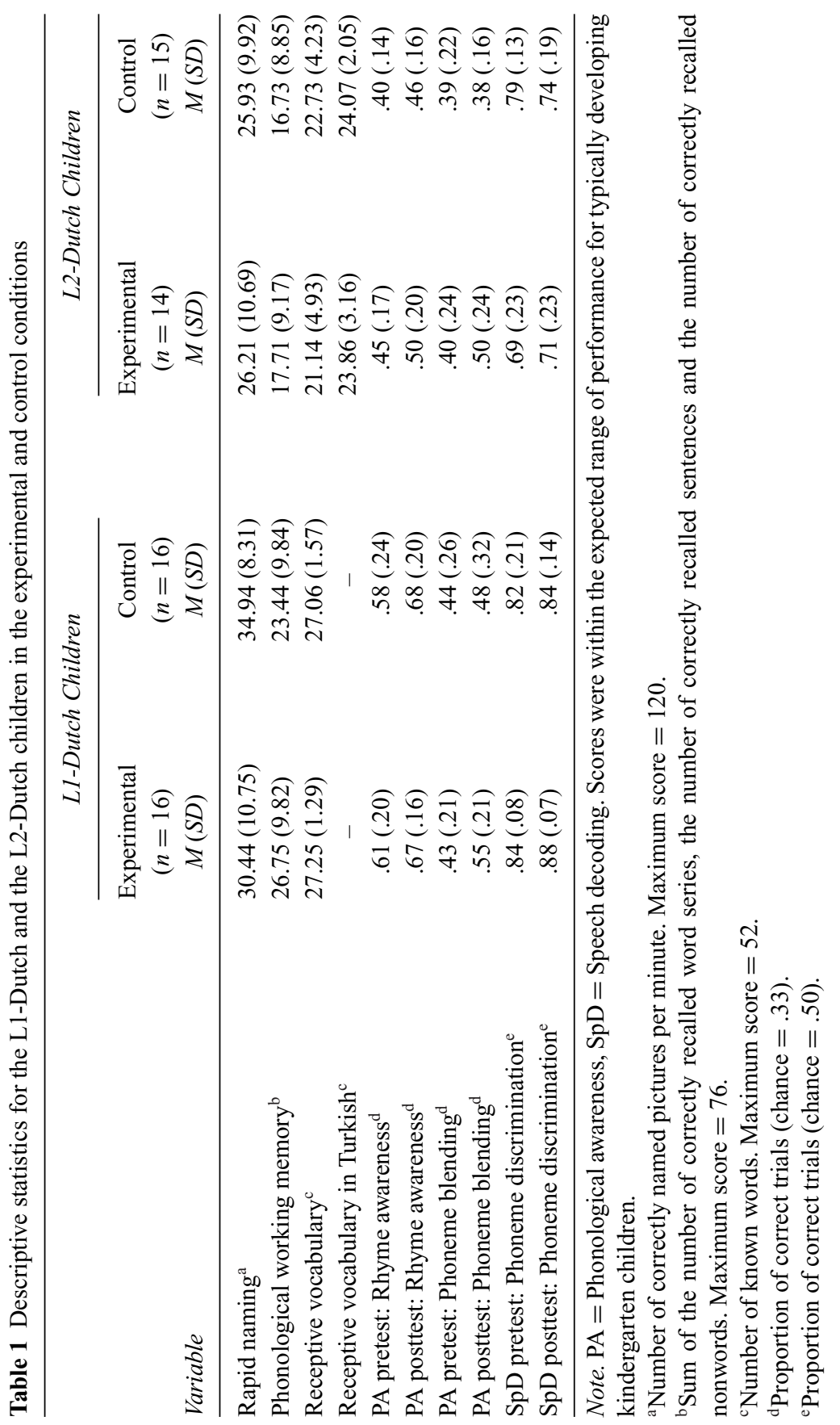


A multivariate analysis of variance (MANOVA) carried out using these measures with group (experimental, control) and language (L1-Dutch, L2Dutch) as between-subjects factors revealed a main effect of language, $F(9,49)$ $=6.74, p<.001, \eta_{p}^{2}=.55$. With educational level of the parents used as a covariate, this main effect of language was still significant, $F(6,39)=3.09$, $p=.014, \eta_{p}^{2}=.32$. No main effect of group or interaction effect between language and group was found (all $F \mathrm{~s}<1$ ). A one-way univariate analysis of variance (ANOVA) comparing Turkish receptive vocabulary scores with group (experimental, control) as a between-subjects factor showed that there was no main effect of group $(F<1)$. The children in the experimental and control groups did not differ in any of the basic cognitive skills or in any of the pretest measures.

The main effect of language suggests that the L1-Dutch and the L2Dutch children differed in some measures. This was further investigated using ANOVAs carried out separately for each measure with language (L1-Dutch, L2-Dutch) as a between-subjects factor. Because the main effect of language remained significant when educational level of the parents was added as a covariate in the MANOVA, this variable was not included as a covariate in the separate ANOVAs for each measure. The L2-Dutch children scored significantly lower than the L1-Dutch children in rapid naming, $F(1,59)=6.79, p=$ $.012, \eta_{p}^{2}=.10$, phonological working memory, $F(1,59)=10.77, p=.002, \eta_{p}^{2}$ $=.15$, receptive Dutch vocabulary, $F(1,59)=38.40, p<.001, \eta_{p}^{2}=.39$, and in the rhyme awareness pretest, $F(1,59)=13.12, p=.001, \eta^{2} p=.18$. There was a trend toward a significant difference in performance on the phoneme discrimination pretest, $F(1,59)=3.74, p=.058, \eta_{p}^{2}=.06$, with the L2-Dutch children scoring lower than the L1-Dutch children. There was no significant difference between L1-Dutch and L2-Dutch children in scores on the phoneme blending pretest, $F(1,59)=.48, p=.493, \eta^{2} p=.01$.

\section{Effects of Lexical Specificity Training}

The first research question was whether lexical specificity training would enhance speech decoding and/or phonological awareness (rhyme awareness and/or phoneme awareness) in bilingual children to the same extent as in their monolingual peers. Repeated-measures ANOVAs were conducted for each of the three experimental measures (rhyme awareness, phoneme blending, and phoneme discrimination scores) with group (experimental, control) and language (L1Dutch, L2-Dutch) as between-subjects factors and time (pretest, posttest) as a within-subjects factor. In the analysis of the rhyme awareness data, a significant main effect of time was found, $F(1,57)=7.03, p=.010, \eta_{p}^{2}=.11$, indicating 


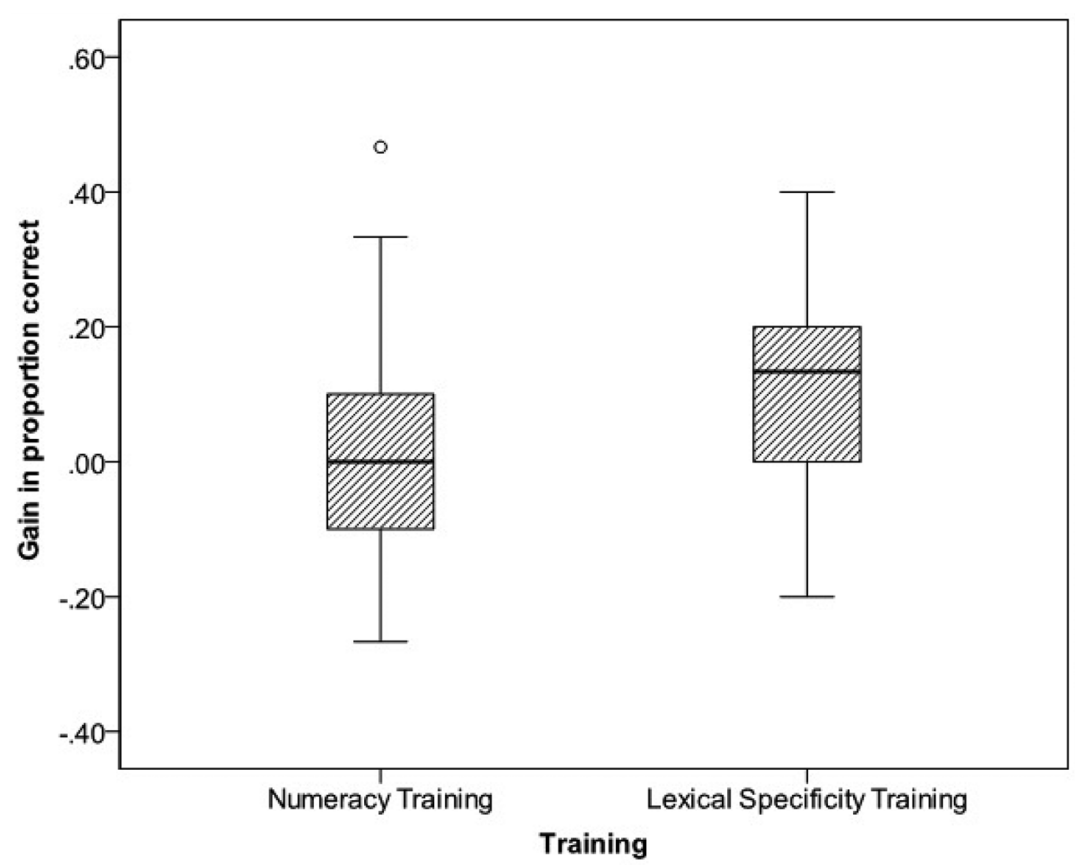

Figure 2 Gains in phoneme blending, defined as posttest minus pretest (proportion correct), for the experimental and control groups. Error bars show $95 \%$ confidence intervals.

an overall improvement. A significant main effect of language was also found, $F(1,57)=20.34, p<.001, \eta_{p}^{2}=.26$. The L1-Dutch children scored higher on rhyme awareness than the L2-Dutch children (see Table 1). There were no other significant main or interaction effects (all $F \mathrm{~s}<1$ ). The experimental group, in other words, did not show more learning gains on rhyme awareness than the control group.

In the analysis of the phoneme blending data, we again found a significant main effect of time, $F(1,57)=9.71, p=.003, \eta_{p}^{2}=.15$, indicating overall improvement, but no significant main effect of language, $F(1,57)=1.05, p=$ $.31, \eta^{2}=.02$. There was also no significant main effect of group, $F(1,57)=$ $.70, p=.41, \eta_{p}^{2}=.01$, but there was a significant time $\times$ group interaction, $F(1,57)=5.11, p=.028, \eta_{p}^{2}=.08$. This interaction reflected a significant increase in phoneme blending for the children in the experimental group, $t(29)$ $=3.96, p<.001, d=.74$, but not for the children in the control group, $t(30)$ $=.63, p=.54, d=.62$, as shown in Figure 2. There were no other significant 
Table 2 Proportion of correct trials in the lexical specificity training for L1-Dutch $(n=$ 16) and L2-Dutch $(n=14)$ participants

\begin{tabular}{|c|c|c|c|c|c|c|}
\hline \multirow[b]{2}{*}{ Phonetic distinction } & \multicolumn{2}{|c|}{ Block 1 and Block 2} & \multirow[b]{2}{*}{$n$} & \multicolumn{2}{|c|}{ Block 3} & \multirow[b]{2}{*}{$n$} \\
\hline & $\begin{array}{c}\text { L1-Dutch } \\
M(S D)\end{array}$ & $\begin{array}{c}\text { L2-Dutch } \\
M(S D)\end{array}$ & & $\begin{array}{l}\text { L1-Dutch } \\
M(S D)\end{array}$ & $\begin{array}{l}\text { L2-Dutch } \\
M(S D)\end{array}$ & \\
\hline $\mathrm{D}$ & $.48(.11)$ & $.33(.09)$ & 48 & $.45(.08)$ & $.33(.12)$ & 12 \\
\hline $\mathrm{D} / \mathrm{T}$ & $.41(.10)$ & $.30(.08)$ & 48 & $.44(.13)$ & $.38(.14)$ & 12 \\
\hline Overall & $.45(.10)$ & $.32(.07)$ & 96 & $.45(.09)$ & $.35(.10)$ & 24 \\
\hline
\end{tabular}

Note. Chance $=.25 . \mathrm{D} / \mathrm{T}=$ minimal phonetic distinctions occurring both in Dutch and Turkish. $\mathrm{D}=$ minimal phonetic distinctions occurring only in Dutch.

interactions. In the analysis of the phoneme discrimination data, no significant main or interaction effects were obtained (all $F \mathrm{~s}<1$ ).

\section{Effects of Phonological Overlap between L1 and L2}

The second research question asked whether there were effects of phonological overlap between L1 and L2 in lexical specificity training for bilingual children (i.e., effects of linguistic transfer). To answer this question, we conducted a series of four one-way ANOVAs targeting the children's performance during the training (proportion of words correctly identified). The four ANOVAs compared: (a) the Dutch-only phonetic distinctions in Blocks 1 and 2, (b) the Dutch/Turkish phonetic distinction in Blocks 1 and 2, (c) the Dutch only phonetic distinction in Block 3, and (d) the Dutch/Turkish phonetic distinction in Block 3. Blocks 1 and 2 needed to be analyzed separately from Block 3 because the target word pairs were presented together only in Block 3. In each ANOVA, each of these four distinctions was the dependent variable, and language (L1-Dutch, L2-Dutch) was always a between-subjects factor. Table 2 shows descriptive statistics for these data.

The analyses revealed significant main effects of language for the Dutchonly phonetic distinctions in Blocks 1 and 2 and in Block 3, with the L1-Dutch children having higher scores than the L2-Dutch children, $F(1,28)=14$.14, $p=.001, d=1.39$, and $F(1,28)=10.25, p=.003, d=1.15$, respectively. A similar effect was found for the Dutch/Turkish phonetic distinctions in Blocks 1 and $2, F(1,28)=10.67, p=.003, d=1.20$, but not anymore in Block 3 . In this final block, the two groups no longer differed in proportion of words identified correctly, $F(1,28)=1.84, p=.19, d=.50$. In other words, the L2-Dutch children narrowed the difference with the L1-Dutch children for the 


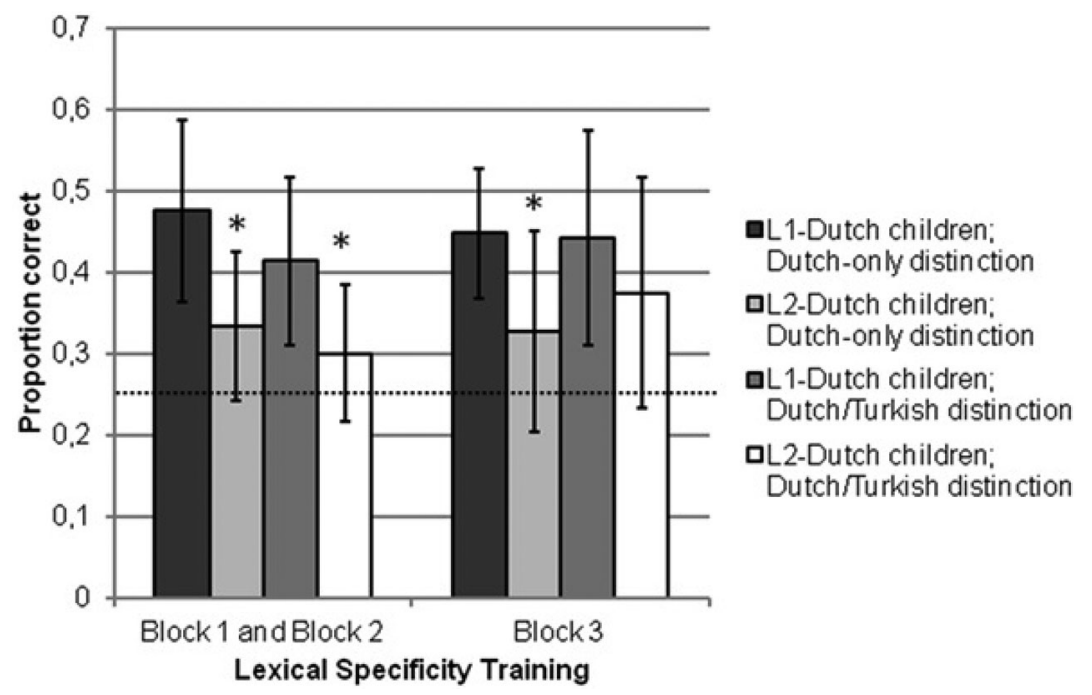

Figure 3 Proportion of correct trials for each phonetic distinction during lexical specificity training. Chance $=.25$. Error bars show $95 \%$ confidence intervals.

Dutch/Turkish phonetic distinctions but not for the Dutch-only distinctions (see Table 2 and Figure 3).

One-sample $t$ tests showed that the L1-Dutch children performed significantly above chance for the Dutch-only distinctions in Blocks 1 and 2, $t(15)=8.00, p<.001$, and in Block 3, $t(15)=9.92, p<.001$, as well as for the Dutch/Turkish distinctions in Blocks 1 and $2, t(15)=6.35, p<.001$, and in Block 3, $t(15)=5.86, p<.001$. Also, the L2-Dutch children performed significantly above chance for the Dutch-only distinctions in Blocks 1 and 2, $t(13)=3.43, p=.005$, and in Block 3, $t(13)=2.33, p=.037$, as well as for the Dutch/Turkish distinctions in Blocks 1 and $2, t(13)=2.25, p=.042$, and in Block 3, $t(13)=3.31, p=.006$.

\section{Discussion}

\section{Summary of Findings}

The present study examined the effects of lexical specificity training targeting speech decoding and phonological awareness in 4-year-old Turkish-Dutch bilingual children (L1 Turkish, L2 Dutch). The role of linguistic transfer was also explored by creating differential overlap between the children's L1 and L2 
in the training materials. The results show that there was significantly more improvement on phoneme blending (an aspect of phonological awareness) for the experimental group than for the control group, in both L1-Dutch and L2-Dutch children, but no effects on rhyme awareness or speech decoding. Second, the difference between the L2-Dutch children and the L1-Dutch children in performance during the lexical specificity training disappeared for the Dutch/Turkish phonetic distinctions in the final (third) block. During the training, the L2-Dutch children seemed to catch up with the L1-Dutch children on the phonetic distinctions that overlapped between the bilingual children's two languages. Lexical specificity training can therefore be effective not only in monolingual children (Van Goch et al., 2014) but also in bilingual children. Despite the bilinguals' lower L2 vocabulary size compared to that of the monolinguals, they were able to learn new L2 words via the training protocol. Critically, phoneme awareness was enhanced in bilingual children after the training.

\section{Lexical Specificity Training and Bilingual Phonological Skills}

It was expected that lexical specificity training would foster speech decoding and phonological awareness. Both the L1-Dutch and the L2-Dutch children showed an improvement only in phoneme blending. In order to perform the phoneme blending task successfully, the correct word as a whole has to be identified based on its individual phonemes (e.g., /d/ / $/$ :/ /r/ has to be identified as deur "door"). Gains in detailed phonological knowledge via lexical specificity training may have fostered phoneme awareness and thus facilitated identification of words in this task (Walley et al., 2003). For both L1-Dutch and L2-Dutch children, lexical specificity training may have stimulated implicit phoneme awareness, because children were confronted with minimal acoustic-phonetic differences among phonemes, rather than larger sound units. This in turn may have generalized to their performance in an explicit phoneme awareness task. For L2-Dutch children, possibly, training lexical specificity in the target language increased their experience with the phonological structure of their L2, but this yielded benefits only in phoneme blending, a task that requires awareness of small sound units (phonemes). According to Campbell and Sais (1995), L2 exposure is particularly beneficial for speech-sound awareness.

The groups did not improve in rhyme awareness due to the training, whereas Van Goch et al. (2014) did find a training effect in this task. Both rhyming and phoneme blending are part of phonological awareness, but rhyming requires awareness of larger sound units than phoneme blending. Van Goch et al. did not assess phoneme blending, but used another task that required 
awareness of smaller sound units, one in which the initial phoneme of a word had to be identified. These researchers found no significant effects of lexical specificity training in this phoneme identification task. A possible explanation for the differences in the outcomes between the two studies relates to the children's age. The children who participated in Van Goch et al.'s (2014) study were on average 4 months younger than the children who participated in the current study. Phonological awareness develops from larger to smaller sound units (Carroll et al., 2003; Vloedgraven \& Verhoeven, 2007; Ziegler \& Goswami, 2005). For example, in a 1-year longitudinal study, Carroll et al. (2003) showed that children gradually improved on a rhyme matching measure from time 1 (mean age 46 months) to time 2 (mean age 50 months) to time 3 (mean age 57 months), whereas children strongly improved on an initial phoneme matching measure only between time 2 and time 3 . These authors suggested that preschool phonological awareness can be divided into an early implicit sensitivity to similarity of sounds and a later explicit awareness of phonemes. The later development of the explicit awareness of smaller sound units appears to build on the foundations of earlier awareness of larger sound units.

It is thus possible that lexical specificity training may most strongly support awareness of the sound units that the children are currently focusing on in their ongoing development of phonological awareness. The older children in the present study may have reached the stage at which they started to become aware of individual phonemes, and hence the training fostered that component of phonological awareness the most, whereas the younger children in Van Goch et al.'s study may have only just begun to become aware of rhymes, and hence the training fostered that component the most. Of course, this does not mean that the older children have fully mastered rhyming skills. In fact, the L1Dutch and the L2-Dutch children, in both the experimental and control groups, improved on rhyme awareness (pre-to-posttest). This overall learning effect is likely due to carryover benefits of learning in a preschool classroom and at home, spontaneous development, and/or test-retest effects. It shows that all aspects of phonological awareness are still developing at this age.

In the current study as well as in the study by Van Goch et al. (2014), lexical specificity training did not impact speech decoding. Possibly, this indicates that lexical specificity mediates between speech decoding and phonological awareness. On this view, development of speech decoding would precede development of lexical specificity, and this would in turn precede the development of phonological awareness. The fact that both the L1-Dutch and the L2-Dutch children performed relatively well on the phoneme discrimination 
pre- and posttests supports this suggestion. However, the null effect on the speech decoding task does not allow any conclusions to be drawn about the exact relationship between speech decoding and lexical specificity. In future research, the relation between these phonological variables should be explored in greater depth.

Our second hypothesis was that similarity between L1 and L2 would promote language transfer and lead to better training results, in turn contributing to enhancement of phonological awareness (MacWhinney, 2004). The results of lexical specificity training point in that direction. The L2-Dutch children performed significantly lower in the training than the L1-Dutch children, but the L2-Dutch children seemed to catch up with the L1-Dutch children on the Dutch phonetic distinctions that were similar to Turkish phonetic distinctions. Possibly, L2-Dutch children were able to better represent the overlapping phonetic distinctions, because these are part of both their L1 and L2. This may have led to faster learning of, and better performance for, words containing these distinctions and may have positively affected training outcomes in bilingual children. This in turn may have contributed to an increase in phoneme blending.

\section{Alternative Explanations}

Several explanations for the results have been discussed based on theoretical grounds. However, in light of the nature of the tasks used in this study, there are other possible explanations. For example, in addition to the specific aspect of phonological awareness assessed by the phoneme blending task, the nature of the task may also have played a role in facilitating phoneme blending for the L2-Dutch children. In a study by Bialystok et al. (2010), only a task with moderate cognitive demands revealed an advantage for bilingual children. Performance on a more challenging task may be primarily dependent on the level of cognitive functioning, whereas an easier task may not be challenging enough to show any effects of bilingualism. In the current study, the rhyme awareness and phoneme discrimination tasks may have been easier for the L1-Dutch children than for the L2-Dutch children and therefore less cognitively demanding for the former group, whereas the phoneme blending task may have been equally challenging for the L1- and L2-Dutch children. In fact, there was no difference in performance between L1-Dutch children and L2-Dutch children on phoneme blending pre- and posttest, whereas there was a clear difference in performance between L1-Dutch and L2-Dutch children on phonological working memory, rapid naming, receptive vocabulary, phoneme discrimination, and rhyme awareness. 
Although the lexical specificity training protocol used in the current study was the same as in the Van Goch et al. (2014) study, there is a possibility that, besides a difference in participant age, a difference in stimulus materials triggered enhancement of different aspects of phonological awareness. In the Van Goch et al. study, only words with minimal phonetic distinctions in consonants were used. Furthermore, in most words, the minimal phonetic distinctions targeted the initial phoneme (16 out of 24 quadruplets), and in the remaining words, the distinctions targeted the final phoneme (8 out of 24 quadruplets). In the current study, words with minimal phonetic distinctions in both consonants and vowels were used, and the distinctions involved initial (8), central (12), and final (4 out of 24 quadruplets) phonemes. More variation in position of the minimal phonetic distinctions in lexical specificity training may have led to improvement in phoneme awareness in the current study, whereas training in Van Goch et al.'s study led to improvement in rhyme awareness.

The design of the lexical specificity training may also provide another explanation for the null effect on the phoneme discrimination task. Although the children were taught words with minimal acoustic-phonetic differences during training, they did not have to discriminate minimal-pair words directly. They had to keep the phonological representation of one word in mind in order to differentiate it from the phonological representation of the word that was asked for. This task may enhance awareness of minimal acoustic-phonetic differences between words at the lexical level, but may not necessarily enhance perception of these differences at the auditory level (i.e., speech decoding skills). Results of a study by Díaz, Mitterer, Broersma, and Sebastián-Gallés (2012) showed that performance of L2 listeners differs depending on the nature of the task. Higher accuracy scores were achieved in an acoustic-phonetic analysis task than in tasks involving lexical processes.

\section{Future Research}

When evaluating the results, a few limitations of the current study have to be taken into account. First, although the degree of phonological overlap between L1 and L2 in stimulus materials appeared to have a positive effect on lexical specificity training results, inferences about the relationship between linguistic transfer and intervention (enhancement of phoneme awareness) can only be drawn tentatively. The effects of lexical specificity training on phoneme blending in bilingual children could be attributed to the training alone or to the training and the overlap between L1 and L2 in stimulus materials combined. As mentioned earlier, lexical specificity training may have 
fostered phoneme awareness, which in turn may have facilitated identification of words in the phoneme blending task. If so, the effects on phoneme blending can be attributed to the lexical specificity training itself. But it is important to remember that, for the L2-Dutch children, the training was administered in their L2. Although this may have increased the children's experience with the target language's phonological structure, this would not necessarily lead to better training outcomes. The interdependency hypothesis (Cummins, 1983, $1984,1991)$ predicts on the one hand that optimal input in one language leads not only to better skills in that language but also to a deeper conceptual and linguistic proficiency that can facilitate the transfer of various cognitive and academic language skills across languages. On the other hand, the hypothesis predicts that L2 learning leads to a lower proficiency in the L1, with near-native proficiency in the L2 almost never reached. So, according to Cummins, experience with two languages can be additive, leading to advantages for bilingual children, or subtractive, causing bilingual children to be disadvantaged with respect to monolingual children. Overlap between L1 and L2 in lexical specificity training may thus boost positive effects of bilingualism or attenuate negative effects. If so, the outcomes of the training may mainly be guided by the similarities between L1 and L2 that are put into training materials. It may therefore be the case that only lexical specificity training in combination with similarities between L1 and L2 in stimulus materials would lead to desirable results. Future research should disentangle effects of each of these two aspects of lexical specificity training in bilingual children.

Second, the children in this study received lexical specificity training only once, for 15 minutes, with only a week between training and posttest. Training that is more in line with teaching methods used in kindergarten may provide more insight into effects of lexical specificity intervention in a classroom setting. Also, given the brevity of the intervention and the nature of the training protocol, as well as the lack of tests within the experimental group targeting changes in lexical specificity of other words outside of the intervention, no claims can be made about the effectiveness of training for the specificity of phonological representations across the lexicon or for fundamental changes in existing linguistic knowledge. Yet, in the experimental group, L1-Dutch children learned, on average, approximately 11 new minimal-pair words, and the L2-Dutch children learned 8 such words (see Table 2), whereas children in the control group did not learn any new minimal pair words during numeracy training. Even if gains in detailed phonological knowledge are limited only to a small number of words, the effect on phoneme blending in the 
experimental group suggests that training in lexical specificity can foster phonological awareness. It would then be interesting to explore effects of similar kinds of training in a larger intervention program.

Third, the process of elimination strategy explained to the children in order to complete the lexical specificity training successfully may not have been the optimal learning approach for the L2-Dutch children. Several studies showed that bilinguals are less likely to use this strategy in word learning than monolinguals, because they are more often confronted with a situation in which two labels apply to one and the same object (e.g., Byers-Heinlein \& Werker, 2009; Houston-Price, Caloghiris, \& Raviglione, 2010). Nevertheless, there are arguments that the overall monolingual-bilingual differences in performance in the current study cannot be accounted for by a difference in the groups' use of the elimination strategy. The strategy was clearly explained before the training started and the children received feedback on their responses during training, cueing them as to which label should be applied to which picture (Houston-Price et al., 2010). Also, when the context makes it clear what language labels come from, 4-year-old children can limit their use of mutual exclusivity appropriately (Au \& Glusman, 1990).

To more deeply explore the results of the current study, future studies may draw from the following ideas. Lexical specificity training with and without phonological overlap between L1 and L2 (with L1- and L2-learning children as participants) may be compared in order to disentangle effects of different aspects of lexical specificity training in bilingual children. Also, L1- and L2-learning children could be trained until they meet the same criterion performance level in lexical specificity training, which might lead to different gains in phonological awareness for L1- and L2-learning children. In both monolingual and bilingual children, the exact relationship among speech decoding, lexical specificity, and phonological awareness, as well as longitudinal effects of lexical specificity training can be examined in a long-term follow-up study in which lexical specificity training is administered several times. Moreover, kindergarten children can be followed until literacy instruction begins, to examine effects of lexical specificity training in literate children. Via classroom intervention studies, it can also be examined how knowledge of lexical specificity and linguistic transfer can be implemented in schools. In the current study, L2-Dutch children were tested solely in their L2 (except for receptive vocabulary, which was tested both in Dutch and Turkish). In order to learn more about the role of L1 knowledge and to study linguistic transfer more extensively, in future studies children could be tested in similar tasks both in their L1 and L2. Finally, more extensive measures of language use and literacy activities 
at home can give more insight into the influence of the home environment on speech decoding, phonological awareness, and the development of lexical specificity.

\section{Conclusion}

The results of the current study show that training designed to make phonological representations for new words more specific can foster phoneme awareness in child L1 and L2 speakers. Differential overlap between L1 and L2 in the targeted words seems to positively affect training outcomes for L2 speakers, likely due to linguistic transfer. This information should be brought into the classroom, where more individualized vocabulary and literacy instruction, depending on the child's linguistic background, could help create the optimal learning environment for each child.

Final revised version accepted 20 June 2014

\section{References}

Au, T. K., \& Glusman, M. (1990). The principle of mutual exclusivity in word learning: To honor or not to honor? Child Development, 61, 1474-1490.

August, D., Snow, C., Carlo, M., Proctor, C. P., deSan Francisco, A. R., Duursma, E., et al. (2006). Literacy development in elementary school second-language learners. Topics in Language Disorders, 26, 351-364.

Best, C. C., \& McRoberts, G. W. (2003). Infant perception of non-native consonant contrasts that adults assimilate in different ways. Language and Speech, 46, 183-216.

Bialystok, E., Luk, G., Peets, K. F., \& Yang, S. J. (2010). Receptive vocabulary differences in monolingual and bilingual children. Bilingualism: Language and Cognition, 13, 525-531.

Bialystok, E., Majumber, S., \& Martin, M. M. (2003). Developing phonological awareness: Is there a bilingual advantage? Applied Psycholinguistics, 24, 27-44.

Bloom, P. (2000). How children learn the meanings of words. Cambridge, MA: MIT Press.

Boeschoten, H. E., \& Verhoeven, L. T. (1987). Language-mixing in children's speech: Dutch language use in Turkish discourse. Language Learning, 37, 191-215.

Byers-Heinlein, K., \& Werker, J. F. (2009). Monolingual, bilingual, trilingual: Infants' language experience influences the development of a word-learning heuristic. Developmental Science, 12, 815-823.

Campbell, R., \& Sais, E. (1995). Accelerated metalinguistic (phonological) awareness in bilingual children. British Journal of Developmental Psychology, 13, 61-68. 
Carroll, D. W. (2008). Psychology of language (5th ed.). London:

Wadsworth/Thomson Learning.

Carroll, J. M., Snowling, M. J., Hulme, C., \& Stevenson, J. (2003). The development of phonological awareness in preschool children. Developmental Psychology, 39, 913-923.

Castles, A., \& Coltheart, M. (2004). Is there a causal link from phonological awareness to success in learning to read? Cognition, 91, 77-111.

Cenoz, J. (2001). Basque in Spain and France. In G. Extra \& D. Gorter (Eds.), The other languages of Europe (pp. 45-57). Clevedon, UK: Multilingual Matters.

Cheour, M., Ceponiene, R., Lehtokoski, A., Luuk, A., Allik, J., Alho, K., et al. (1998). Development of language-specific phoneme representations in the infant brain. Nature Neuroscience, 1, 351-353.

Cummins, J. (1983). Heritage language education: A literature review. Toronto: Ministry of Education.

Cummins, J. (1984). Wanted: A theoretical framework for relating language proficiency to academic achievement among bilingual students. In C. Rivera (Ed.), Language proficiency and academic achievement (pp. 2-19). Clevedon, UK: Multilingual Matters.

Cummins, J. (1991). Conversational and academic language proficiency in bilingual contexts. In J. H. Hulstijn \& J. F. Matter (Eds.), Reading in two languages: AILA review. Amsterdam: Free University Press.

deBot, K. (1992). A bilingual production model: Levelt's "speaking" model adapted. Applied Linguistics, 13, 1-24.

DeCara, B., \& Goswami, U. (2003). Phonological neighbourhood density: Effects in a rhyme awareness task in five-year-old children. Journal of Child Language, 30, 695-710.

Díaz, B., Mitterer, H., Broersma, M., \& Sebastián-Gallés, N. (2012). Individual differences in late bilinguals' L2 phonological processes: From acoustic-phonetic analysis to lexical access. Learning and Individual Differences, 22, 680-689.

Dickinson, D. K., McCabe, A., Clark-Chiarelli, N., \& Wolf, A. (2004). Cross-language transfer of phonological awareness in low-income Spanish and English bilingual preschool children. Applied Psycholinguistics, 25, 323-347.

Elbro, C., Borstrøm, I., \& Petersen, D. K. (1998). Predicting dyslexia from kindergarten: The importance of distinctness of phonological representations of lexical items. Reading Research Quarterly, 33, 36-60.

Francis, N. (2005). Research findings on early first language attrition: Implications for the discussion on critical periods in language acquisition. Applied Linguistics, 55, 491-531.

Garlock, V. M., Walley, A. C., \& Metsala, J. L. (2001). Age-of-acquisition, word frequency, and neighborhood density effects on spoken word recognition by children and adults. Journal of Memory and Language, 45, 468-492. 
Gerken, L. A., Murphy, W. D., \& Aslin, R. N. (1995). Three- and four-year-olds' perceptual confusions for spoken words. Perception and Psychophysics, 57, 475-486.

Gildersleeve-Neumann, C. E., Pena, E. D., Davis, B. L., \& Kester, E. S. (2009). Effects on L1 during early acquisition of L2: Speech changes in Spanish at first English contact. Bilingualism: Language and Cognition, 12, 259-272.

Goldstein, B. A., \& Bunta, F. (2012). Positive and negative transfer in the phonological systems of bilingual speakers. International Journal of Bilingualism, 16, 388-401.

Goswami, U. (2000). Phonological representations, reading development and dyslexia: Towards a cross-linguistic theoretical framework. Dyslexia, 6, 133-151.

Gottardo, A., Yan, B., Siegel, L., \& Wade-Woolley, L. (2001). Factors related to English reading performance in children with Chinese as a first language: More evidence of cross-language transfer of phonological processing. Journal of Educational Psychology, 93, 530-542.

Grosjean, F. (2012). An attempt to isolate, and then differentiate, transfer and interference. International Journal of Bilingualism, 16, 11-21.

Hernandez, A., Li, P., \& MacWhinney, B. (2005). The emergence of competing modules in bilingualism. Trends in Cognitive Sciences, 9, 220-225.

Houston-Price, C., Caloghiris, Z., \& Raviglione, E. (2010). Language experience shapes the development of the mutual exclusivity bias. Infancy, 15, 125-150.

$\mathrm{Hu}, \mathrm{C}$. (2003). Phonological memory, phonological awareness, and foreign language word learning. Language Learning, 53, 429-462.

Jarvis, S., \& Odlin, T. (2000). Morphological type, spatial reference, and language transfer. Studies in Second Language Acquisition, 22, 535-556.

Jia, G., Strange, W., Wu, Y, Collado, J., \& Guan, Q. (2006). Perception and production of English vowels by Mandarin speakers: Age-related differences vary with amount of L2 exposure. Journal of the Acoustical Society of America, 119, 1118-1130.

Kan, P. F. (2010). Measuring word learning ability in sequential bilingual children. Perspectives on Communication Disorders and Sciences in Culturally and Linguistically Diverse Populations, 17, 25-32.

Kan, P. F., \& Kohnert, K. (2005). Preschoolers learning Hmong and English: Lexical semantic skills in L1 and L2. Journal of Speech, Language, and Hearing Research, 48, 372-383.

Kan, P. F., \& Kohnert, K. (2008). Fast mapping by developing bilinguals in L1 and L2. Journal of Child Language, 35, 495-514.

Kan, P. F., \& Kohnert, K. (2012). A growth curve analysis of novel word learning by sequential bilingual preschool children. Bilingualism: Language and Cognition, 15, 452-469.

Kieffer, M. J. (2008). Catching up or falling behind? Initial English proficiency, concentrated poverty, and the reading growth of language minority learners in the United States. Journal of Educational Psychology, 100, 851-868. 
Kohnert, K. J., \& Bates, E. (2002). Balancing bilinguals II: Lexical comprehension and cognitive processing in children learning Spanish and English. Journal of Speech Language and Hearing Research, 45, 347-359.

Kohnert, K., Windsor, J., \& Ebert, K. D. (2009). Primary or "specific" language impairment and children learning a second language. Brain and Language, 109, 101-111.

Kuhl, P. K. (2004). Early language acquisition: Cracking the speech code. Nature Reviews Neuroscience, 5, 831-843.

Leseman, P. P. M. (2000). Bilingual vocabulary development of Turkish preschoolers in the Netherlands. Journal of Multilingual and Multicultural Development, 21, 93-112.

MacWhinney, B. (2004). A unified model of language acquisition. In J. F. Kroll \& A. M. B. deGroot (Eds.), Handbook of bilingualism: Psycholinguistic approaches (pp. 49-67). Oxford, UK: Oxford University Press.

Mark, S., Müller-Myhsok, B., Schulte-Körne, G., \& Landerl, K. (2014). Cognitive mechanisms underlying reading and spelling development in five European orthographies: Is English an outlier orthography? Learning and Instruction, 29, $65-77$.

Melby-Lervåg, M., Halaas Lyster, S. A., \& Hulme, C. (2012). Phonological skills and their role in learning to read: A meta-analytic review. Psychological Bulletin, 138, $322-352$.

Metsala, J. L. (1999). Young children's phonological awareness and nonword repetition as a function of vocabulary development. Journal of Educational Psychology, 91, $3-19$.

Metsala, J. L., \& Walley, A. C. (1998). Spoken vocabulary growth and the segmental restructuring of lexical representations: Precursors to phonemic awareness and early reading ability. In J. L. Metsala \& L. C. Ehri (Eds.), Word recognition in beginning literacy (pp. 89-120). Mahwah, NJ: Erlbaum.

Morais, J. (2003). Levels of phonological representation in skilled reading and in learning to read. Reading and Writing: An Interdisciplinary Journal, 16, 123-151.

Mulder, F., Timman, Y., \& Verhallen, S. (2009). Basiswoordenlijst Amsterdamse Kleuters [Basic Vocabulary of Kindergartners in Amsterdam]. Amsterdam: Drukkerij De Raat \& De Vries.

Muter, V., Hulme, C., Snowling, M. J., \& Stevenson, J. (2004). Phonemes, rimes, vocabulary, and grammatical skills as foundations of early reading development: Evidence from a longitudinal study. Developmental Psychology, 40, 665-681.

Navarra, J., Sebastián-Gallés, N., \& Soto-Faraco, S. (2005). The perception of second language sounds in early bilinguals: New evidence from an implicit measure. Journal of Experimental Psychology, 31, 912-918.

Odlin, T. (1989). Language transfer: Cross-linguistic influence in language learning. Cambridge, UK: Cambridge University Press. 
Patel, T. K., Snowling, M. J., \& deJong, P. F. (2004). A cross-linguistic comparison of children learning to read in English and Dutch. Journal of Educational Psychology, 96, 785-797.

Poulisse, N. (1990). The use of compensatory strategies by Dutch learners of English. Dordrecht, Netherlands: Foris.

Roberts, T. A. (2005). Articulation accuracy and vocabulary size contributions to phonemic awareness and word reading in English language learners. Journal of Educational Psychology, 97, 601-616.

Schaerlaekens, A., Kohnstamm, D., Lejaegere, M., deVries, A., Peeters, L., \& Zink, L. (1999). Streeflijst Woordenschat voor 6-jarigen [Target List for 6-year-olds]. Lisse, Netherlands: Swets \& Zeitlinger.

Selinker, L., \& Lakshmanan, U. (1993). Language transfer and fossilization: The "Multiple Effects Principle." In S. Gass \& L. Selinker (Eds.), Language transfer in language learning (rev. ed., pp. 197-216). Amsterdam: John Benjamins.

Siok, W. T., \& Fletcher, P. (2001). The role of phonological awareness and visual-orthographic skills in Chinese reading acquisition. Developmental Psychology, 37, 886-899.

Swingley, D., \& Aslin, R. N. (2000). Spoken word recognition and lexical representation in very young children. Cognition, 76, 147-166.

Thiessen, E. D. (2007). The effect of distributional information on children's use of phoneme contrasts. Journal of Memory and Language, 56, 16-34.

Torgesen, J. K., Morgan, S. T., \& Davis, C. (1992). Effects of two types of phonological awareness training on word learning in kindergarten children. Journal of Educational Psychology, 84, 364-370.

Treiman, R., \& Zukowski, A. (1996). Children's sensitivity to syllables, onsets, rimes, and phonemes. Journal of Experimental Child Psychology, 61, 193-215.

Uchikoshi, Y. (2006). English vocabulary development in bilingual kindergarteners: What are the best predictors? Bilingualism-Language and Cognition, 9, 33-49.

VanGoch, M. M., McQueen, J. M., \& Verhoeven, L. (2014). Learning phonologically specific new words fosters rhyme awareness in Dutch preliterate children. Scientific Studies of Reading, 18, 155-172.

Verhoeven, L. (2005). Screeningstest voor Taal- en Leesproblemen [Diagnostic Test for Language and Literacy Problems]. Arnhem, Netherlands: Cito.

Verhoeven, L. (2007). Early bilingualism, language transfer, and phonological awareness. Applied Psycholinguistics, 28, 425-439.

Verhoeven, L., Narain, G., Extra, G., Konak, O. A., \& Zerrouk, R. (1995). Toets Tweetaligheid [Test Bilingualism]. Arnhem, Netherlands: Cito.

Verhoeven, L., \& Vermeer, A. (2006a). Sociocultural variation in literacy achievement. British Journal of Educational Studies, 54, 189-211.

Verhoeven, L., \& Vermeer, A. (2006b). Taaltoets Alle Kinderen [Language Test for all Children]. Arnhem, Netherlands: Cito. 
Vloedgraven, J. M., Keuning, J., \& Verhoeven, L. (2009). Screeningsinstrument Beginnende Geletterdheid [Screening Instrument for Emerging Literacy]. Arnhem, Netherlands: Cito.

Vloedgraven, J. M., \& Verhoeven, L. (2007). Screening of phonological awareness in the early elementary grades: An IRT approach. Annals of Dyslexia, 57, 33-50.

Walley, A. C., Metsala, J. L., \& Garlock, V. M. (2003). Spoken vocabulary growth: Its role in the development of phoneme awareness and early reading ability. Reading and Writing, 16, 5-20.

Werker, J. F., Byers-Heinlein, K., \& Fennell, C. T. (2009). Bilingual beginnings to learning words. Philosophical Transactions of the Royal Society B: Biological Sciences, 364, 3649-3663.

White, K. S., \& Morgan, J. L. (2008). Sub-segmental detail in early lexical representations. Journal of Memory and Language, 59, 114-132.

Yoshida, K. A., Fennell, C. T., Swingley, D., \& Werker, J. F. (2009). Fourteen-monthold infants learn similar-sounding words. Developmental Science, 12, 412-418.

Ziegler, J., \& Goswami, U. (2005). Reading acquisition, developmental dyslexia, and skilled reading across languages: A psycholinguistic grain size theory.

Psychological Bulletin, 131, 3-29.

\section{Supporting Information}

Additional Supporting Information may be found in the online version of this article at the publisher's website:

Appendix S1: Lexical Specificity Training Stimulus Materials

Appendix S2. Experimental Design and Examples 